Session 2793

\title{
Optimum Matching of Two Unequal Impedances in Acoustical, Optical, or Microwave Stepped Transmission-Lines
}

\author{
Albert W. Biggs \\ University of Alabama at Huntsville
}

\begin{abstract}
A simplified method is described for designing periodic structures with transformer sections connected in series to match impedances or provide transitions from slow wave to fast wave transmission lines in acoustical, optical, and microwave waveguides. For a given bandwidth, transformer section characteristic impedances are designed to provide a Chebyshev Polynomial, or equal ripple, reflection coefficient response, which reduces reflections to negligible levels. It extends Dolph-Chebyshev antenna theory to transmission line transformers. The earlier method was W.W. Hansen's Binomial Coefficient design for several transformer sections. Over a bandwidth ratio of $\mathbf{f}_{\mathbf{2}} / \mathbf{f}_{\mathbf{1}}=\mathbf{2 . 0}$, the Chebyshev Polynomial method has a VSWR of 1.02 to the binomial coefficient design with a VSWR of 1.13, for a line with five sections. Chebyshev polynomials are tedious to calculate, but an unwritten method, developed by Ross E. Graves at Stanford University, makes the calculations as simple as those in Pascal's Triangle for Binomial Coefficients. My thesis advisors, Donald Reynolds and Myron Swarm at Stanford, were students under Professor Graves, and enjoyed my reference to Graves Pyramid for Chebyshev Polynomials.
\end{abstract}

\section{Introduction}

In microwave and optical waveguides, phase velocities are infinite at the cutoff frequency and are always greater than the velocity of light in the dielectric in the guide, where group velocity is zero at cutoff and is always less than the velocity of light in the dielectric in the guide. As the frequency increases far beyond cutoff, phase and group velocities both approach the velocity of light in the dielectric in metallic and dielectric guides. These phase velocities are described as fast waves. In traveling wave tubes, slow wave structures create phase velocities with velocities along the axis of the structure much less than the velocity of light. In slow wave structures such as traveling wave tubes, acoustical horns, and optical telephone circuits, it is necessary to couple these lines to fast wave structures for purposes of transmitting data or radiating electromagnetic and acoustical waves.

This paper describes designing transformer structures for matching acoustical and electromagnetic wave transmission lines with different characteristic impedances, and fast or slow wave

Proceedings of the 2001 American Society for Engineering Education Annual Conference \&Exposition Copyright $O$ 2001, American Society for Engineering Education 
lines with different phase velocities. Design techniques were formerly developed by Seymour Cohn ${ }^{1}$ for fast wave lines like those in waveguides and antennas. This study modifies Cohn's techniques for coupling slow wave to fast wave lines. The transformer structure is a sequence of sections, each with the same acoustical, microwave, or optical length, slowly changing characteristic impedances from section to section, and slightly increasing phase velocities than the preceding section. These gradually increasing slow phase velocity sections may be coaxial, waveguide, or parallel planes. They may be dielectric filled metallic waveguides; dielectric clad optical lines, or acoustical channels. With a given number of sections, the modified method yields maximum bandwidth for a given VSWR, or minimum VSWR for a given bandwidth. It is called a Chebyshev transformer because Chebyshev polynomials are used in its formulation.

\section{Binomial Transformers}

The accepted method for designing transformer structures was Hansen's Binomial coefficient ${ }^{2}$ design prior to development of the Chebyshev or optimum stepped transformer. The Binomial transformer is obtained from the Chebyshev transformers for unity bandwidth ratios.

Maximally flat passband filter responses are obtained where the reflection coefficient $\rho(\mathrm{M})$, between sections $\mathrm{M}$ and $\mathrm{M}+1$ in the multisection transmission line, are proportional to the Binomial coefficients, like those in Binomial or Butterworth filters. This requires that the first $(\mathrm{N}-1)$ derivatives (with respect to $\theta$ ) of the reflection coefficient $\Gamma$ vanish at the matching frequency where $\beta l=\theta=\pi / 2$. These are realized with the function

$$
\Gamma=A\left(1+e^{-j 2 \theta}\right)^{N-1}
$$

and to find the arbitrary constant $A$, the magnitude of $\Gamma$ is written as the function $\rho$,

$$
\rho=|\Gamma|=\left|\mathrm{A} 2^{\mathrm{N}-1}(\cos \theta)^{\mathrm{N}-1}\right|,
$$

and where $\mathrm{N}$ is the number of transformer sections. The constant $\mathrm{A}$ is found at $\theta=0$ or $\pi$, where

$$
\begin{aligned}
& \mathrm{A}\left(2^{\mathrm{N}-1}\right)=\frac{\mathrm{Z}(\mathrm{N}+1)-\mathrm{Z}(1)}{\mathrm{Z}(\mathrm{N}+1)+\mathrm{Z}(1)}, \\
& A=2^{-(N-1)} \frac{Z(N+1)-Z(1)}{Z(N+1)+Z(1) .}
\end{aligned}
$$

where $\mathrm{Z}(1)$ is the characteristic impedance of the input transmission line and $\mathrm{Z}(\mathrm{N}+1)$ of the output line, and the reflection coefficient expression for media with these characteristic impedances, $[\mathrm{Z}(\mathrm{N}+1)-\mathrm{Z}(1)] /[\mathrm{Z}(\mathrm{N}+1)+\mathrm{Z}(1)]$. 
With A from Eq. (3), Eq. (1) is expanded by the Binomial Theorem to obtain the Binomial coefficients,

$$
\begin{aligned}
& \Gamma=2^{-(\mathrm{N}-1)}[\mathrm{Z}(\mathrm{N}+1)+\mathrm{Z}(1)] /[\mathrm{Z}(\mathrm{N}+1)+\mathrm{Z}(1)]\left(1+\mathrm{e}^{-\mathrm{j} 2 \theta}\right)^{\mathrm{N}-1} \\
& =2^{-(\mathrm{N}-1)}[\mathrm{Z}(\mathrm{N}+1)+\mathrm{Z}(1)] /[\mathrm{Z}(\mathrm{N}+1)+\mathrm{Z}(1)]\left\{1+(\mathrm{N}-1) \mathrm{e}^{-\mathrm{j} 2 \theta}+\frac{(\mathrm{N}-1)(\mathrm{N}-2)}{2 !} \mathrm{e}^{\mathrm{j} \theta}+\right. \\
& \ldots .+\frac{(N-1)(N-2) \ldots . .(N-M+1)}{(M-1) !} e^{-j 2(M-1) \theta}+\ldots .+\frac{(N-1)(N-2)}{2 !} e^{-j 2(N-3) \theta} \\
& \left.+(\mathrm{N}-1) \mathrm{e}^{-\mathrm{j} 2(\mathrm{~N}-2) \theta}+\mathrm{e}^{-\mathrm{j} 2(\mathrm{~N}-1) \theta}\right\} \\
& =2^{-(N-1)} \frac{\underline{Z}(N+1)-Z(1)}{Z(N+1)+Z(1)} \sum_{M=1}^{M=N} \frac{(N-1)(N-2) \ldots . .(N-M+1)}{(M-1) !} \quad e^{-j 2(M-1) \theta} \\
& =2^{-(\mathrm{N}-1)} \frac{\underline{\mathrm{Z}(\mathrm{N}+1)-\mathrm{Z}(1)}}{\mathrm{Z}(\mathrm{N}+1)+\mathrm{Z}(1)} \sum_{\mathrm{M}=1}^{\mathrm{M}=\mathrm{N}}|\mathrm{N}-1| \\
& =\quad \sum_{M=1}^{M=N} \rho(M) e^{-j 2(M-1) \theta},
\end{aligned}
$$

where the Binomial coefficients $|\mathrm{M}-1|$ are equal to

$$
\frac{(\mathrm{N}-1)(\mathrm{N}-2) \ldots(\mathrm{N}-\mathrm{M}+1)}{(\mathrm{M}-1) !}=\frac{(\mathrm{N}-1) !}{(\mathrm{N}-\mathrm{M}) !(\mathrm{M}-1) !}=|\mathrm{N}-1| \mathrm{N}-\mathrm{M} \mid,
$$

and where the reflection coefficient $\rho(\mathrm{M})$, between sections $\mathrm{M}$ and $\mathrm{M}+1$, is seen to be proportional to these Binomial coefficients,

$$
\begin{aligned}
\rho(\mathrm{M}) & =[\mathrm{Z}(\mathrm{M}+1)-\mathrm{Z}(\mathrm{M})] /[\mathrm{Z}(\mathrm{M}+1)+\mathrm{Z}(\mathrm{M})] \\
& =2^{-(\mathrm{N}-1)}|\mathrm{N}-1| \mathrm{M} \mid[\mathrm{Z}(\mathrm{N}+1) \mathrm{Z}(1)][\mathrm{Z}(\mathrm{N}+1)+\mathrm{Z}(1)] .
\end{aligned}
$$

Another form is made in a logarithmic form with the series,

$\ln X=2 \frac{|X-1|}{|X+1|}+\frac{2}{3} \frac{|X-1|^{2}}{|X+1|}+\ldots .+\frac{2}{(2 M+1)}{\stackrel{|X-1|^{2}}{|X+1|}}^{\mid X+\ldots . .}=2 \mid \underline{X+1 \mid}+$

for $M=1.2,3, \ldots$ for small values of $X$. Results are within $9 \%$ for $X=3$, and $3 \%$ for $X=2$.

Proceedings of the 2001 American Society for Engineering Education Annual Conference \&Exposition Copyright O 2001, American Society for Engineering Education 
With $X=Z(M+1) / Z(1)$, symmetry gives expressions for section reflection coefficients $\rho(M)$,

$$
\begin{aligned}
& \rho(\mathrm{M})=2^{-(\mathrm{N}-1)}|\mathrm{N}-1| \\
&|\mathrm{N}-\mathrm{M}|[\mathrm{Z}(\mathrm{N}+1)-\mathrm{Z}(1)] /[\mathrm{Z}(\mathrm{N}+1)+\mathrm{Z}(1)] \\
&=2^{-\mathrm{N}}|\mathrm{N}-\mathrm{N}-\mathrm{M}| \ln |\mathrm{Z}(\mathrm{N}+1) / \mathrm{Z}(1)|
\end{aligned}
$$

or in terms of Eq. (6), $\quad \rho(\mathrm{M})=1 / 2 \ln |\mathrm{Z}(\mathrm{M}+1) / \mathrm{Z}(\mathrm{M})|$,

and again, the reflection coefficients again are proportional to the Binomial coefficients and a constant.

\section{Pascal's Triangle and the Binomial Coefficients}

The Binomial coefficients can be calculated for the section reflection coefficients $\rho(\mathrm{M})$ with a simple process. Like the Graves' Pyramid, Pascal's Triangle permits a very simple method of calculation. Table I is Pascal's Triangle for Binomial coefficients.

Table I

Pascal's Triangle for Binomial Coefficients

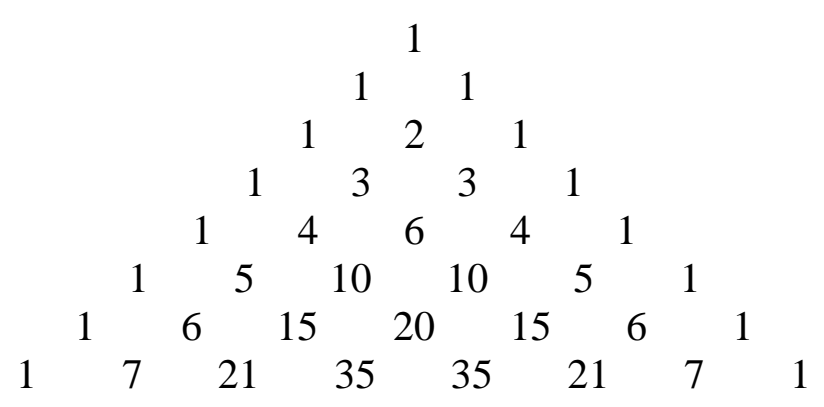

Pascal's triangle is made with the number 1.0 in the first row. In the second row, 1.0s are placed at the left and right of the element above. In successive rows, elements are formed by adding the two elements on the left and right of the calculated element in the row above the element.

With Pascal's Triangle, the ratios of reflection coeffients to design a Binomial transformer for coupling slow to fast wave transmission lines for three steps are

$$
\rho(1): \rho(2): \rho(3)=1.0: 2.0: 1.0,
$$

and for four steps $\rho(1): \rho(2): \rho(3): \rho(4)=1.0: 3.0: 3.0: 1.0$, and in the same manner for $\mathrm{N}$ steps. The results were applied to optical and microwave coupling 
transitions from slow to fast wave lines.

\section{Chebyshev's Transformers}

Hansen's Binomial coefficient design ${ }^{2}$ for transmission line transformer sections was described in the preceding sections. In his design, the logarithms of the characteristic impedance ratios, or the reflection coefficients, of adjacent sections were made to be in the ratio of Binomial coefficients. In this section, the design method calculates the logarithms of the characteristic impedance ratios so that the VSWRs have the characteristic "equal ripple" response of a Chebyshev polynomial. Instead of the maximally flat passband characteristic, the Chebyshev transformer provides a variation of reflection coefficient $\rho$ to vary or oscillate between 0 and $\rho(M)$ across the passband like a Chebyshev filter. Since the equal ripple response makes $\rho$ behave like a Chebyshev polynomial, it is named the Chebyshev transformer. The reflection is zero at as many different frequencies in the passband as there are transformer sections.

Use of Chebyshev polynomials $\mathrm{T}_{\mathrm{N}-1}(\mathrm{z})$ of $(\mathrm{N}-1)$ th degree and of the first kind by looking at some definitions and forms,

$$
\begin{gathered}
\mathrm{T}_{0}(\mathrm{z})=1 \\
\mathrm{~T}_{1}(\mathrm{z})=\mathrm{z} \\
\mathrm{T}_{2}(\mathrm{z})=2 \mathrm{z}^{2}-1 \\
\mathrm{~T}_{3}(\mathrm{z})=4 \mathrm{z}^{3}-3 \mathrm{z} \\
\mathrm{T}_{4}(\mathrm{z})=8 \mathrm{z}^{4}-8 \mathrm{z}^{2}+1 \\
\mathrm{~T}_{5}(\mathrm{z})=16 \mathrm{z}^{5}-20 \mathrm{z}^{3}+5 \mathrm{z} \\
\cdots \cdots \\
\mathrm{T}_{\mathrm{M}}(\mathrm{z})=2 \mathrm{z} \mathrm{T}_{\mathrm{M}-1}(\mathrm{z})-\mathrm{T}_{\mathrm{M}-2}(\mathrm{z})
\end{gathered}
$$

$\mathrm{T}_{\mathrm{M}}(\mathrm{z})$ is also found from the equivalent expressions,

$$
\begin{gathered}
\mathrm{T}_{\mathrm{M}}(\mathrm{z})=\cos \left(\mathrm{M} \cos ^{-1} \mathrm{z}\right),|\mathrm{z}| \leqq 1 \\
\mathrm{~T}_{\mathrm{M}}(\mathrm{z})=\cosh \left(\mathrm{M} \cosh ^{-1} \mathrm{z}\right),|\mathrm{z}| \geqq 1 .
\end{gathered}
$$

When $\mathrm{z}=\cos \theta$, another for $\mathrm{T}_{\mathrm{M}}(\mathrm{z})$ used in Chebyshev transformers is

$$
\mathrm{T}_{\mathrm{M}}(\mathrm{z})=\cos (\mathrm{Mz}) .
$$

Chebyshev polynomials all oscillate between \pm 1 for $z$ between \pm 1 and $\left|T_{M}(z)\right|$ increase monotonically for $|z| \geqq 1$.

In multisection transformers with small reflections at each boundary, like those in previous sections, the overall reflection coefficient is the sum of smaller reflections at each junction, 


$$
\begin{aligned}
\Gamma= & \rho(1)+\rho(2) e^{-j 2 \theta}+\rho(3) e^{-j 4 \theta}+\ldots .+\rho(N) e^{-j 2(N-1) \theta} \\
= & e^{-j(N-1) \theta}\left[\rho(1) e^{j(N-1) \theta}+\rho(2) e^{j(N-3) \theta}+\rho(3) e^{j(N-5) \theta}+\ldots \ldots\right. \\
& \left.\quad+\rho(M) e^{j(N-2 M+1) \theta}+\ldots .+\rho(N-1) e^{-j(N-3) \theta}+\rho(N) e^{-j(N-1) \theta}\right]
\end{aligned}
$$

and the magnitude of the reflection coefficient $\rho$, referred to the center, is given by

$$
\begin{aligned}
\rho=|\Gamma|= & \rho(1) e^{j(N-1) \theta}+\rho(2) e^{j(N-3) \theta}+\rho(3) e^{j(N-5) \theta}+\ldots .+\rho(M) e^{j(N-2 M+1) \theta} \\
& +\ldots .+\rho(N-1) e^{-j(N-3) \theta}+\rho(N) e^{-j(N-1) \theta}
\end{aligned}
$$

The reflection coefficients $\rho(\mathrm{M})$ at each section are assumed to be symmetrical as in the case of the Binomial transformer so that $\rho(1)=\rho(\mathrm{N}), \rho(2)=\rho(\mathrm{N}-1)$, etc., and with this symmetry, the above expression for odd $\mathrm{N}, \mathrm{N}=1,3,5, \ldots$, becomes

$$
\begin{aligned}
\rho=2 \rho(1) \cos (\mathrm{N}-1) \theta+ & 2 \rho(2) \cos (\mathrm{N}-3) \theta+2 \rho(3) \cos (\mathrm{N}-5) \theta+\ldots . . \\
& +2 \rho(\mathrm{M}) \cos (\mathrm{N}-2 \mathrm{M}+1) \theta+\ldots . .+([\mathrm{N}+1] / 2),
\end{aligned}
$$

and for even $\mathrm{N}, \mathrm{N}=2,4,6, \ldots$, becomes

$$
\begin{aligned}
\rho=2 \rho(1) \cos (\mathrm{N}-1) & \theta+2 \rho(2) \cos (\mathrm{N}-3) \theta+2 \rho(3) \cos (\mathrm{N}-5) \theta+\ldots . \\
& +2 \rho(\mathrm{M}) \cos (\mathrm{N}-2 \mathrm{M}+1) \theta+\ldots+2 \rho(\mathrm{N} / 2) \cos \theta .
\end{aligned}
$$

In order to achieve optimum equal ripple response of a Chebyshev filter or transformer, the reflection coefficients $\rho(\mathrm{M})$ must be chosen so that the total reflection coefficient $\rho$ will be proportional to a Chebyshev polynomial. These were defined earlier in this section. The properties of Chebyshev polynomials are that they all oscillate between \pm 1 for $z$ between \pm 1 and $\left|\mathrm{T}_{\mathrm{M}}(\mathrm{z})\right|$ increases monotonically for $|\mathrm{z}| \geqq 1$.

The optimum section reflection coefficient $\rho(\mathrm{M})$ response is obtained by expressing the trigonometric terms below in Eqs. (18) and (19),

$$
\begin{aligned}
& \mathrm{T}_{0}(\cos \theta)=1 \\
& \cos \theta=\mathrm{T}_{1}(\cos \theta)=\mathrm{Y} \\
& \cos 2 \theta=\mathrm{T}_{2}(\cos \theta)=2 \mathrm{Y}^{2}-1 \\
& \cos 3 \theta=\mathrm{T}_{3}(\cos \theta)=4 \mathrm{Y}^{3}-3 \mathrm{Y} \\
& \cos 4 \theta=\mathrm{T}_{4}(\cos \theta)=8 \mathrm{Y}^{4}-8 \mathrm{Y}^{2}+1 \\
& \cos 5 \theta=\mathrm{T}_{5}(\cos \theta)=16 \mathrm{Y}^{5}-20 \mathrm{Y}^{3}+5 \mathrm{Y}
\end{aligned}
$$




$$
\cos \mathrm{M} \theta=\mathrm{T}_{\mathrm{M}}(\cos \theta)=2 \mathrm{Y} \mathrm{T}_{\mathrm{M}-1}(\cos \theta)-\mathrm{T}_{\mathrm{M}-2}(\cos \theta)
$$

Then equate

$$
\mathrm{Y}=\cos \theta=\mathrm{X} \cos \theta_{1}
$$

Equation (19) requires that $\mathrm{X} \leqq 1$ for $\theta_{1}$ and $\theta_{2}$, where $\theta_{1}$ is the electrical spacing at the lower edge of the electrical spacing at the lower edge of the band, and $\theta_{2}=180^{\circ}-\theta_{1}$ is the upper edge of the band.. In this symmetrical range of $\theta$, the reflection coefficient is kept low. The angle $\theta_{1}$ may have any value between 0 and 90 degrees. If $\theta_{1}$ is 60 degrees, then the bandwidth ratio $\mathrm{BW}, \theta_{2} / \theta_{1}$, is 2.00. In the Binomial transformer, $\theta_{1}$ is $90^{\circ}$, so that $\mathrm{BW}$ is one, so that the Binomial transformer is a special case of the Chebyshev transformer

After substituting Eqs. (19) and (20) into Eqs. (18) and (19), polynomials of either odd or even powers of $\mathrm{X}$ are obtained. When a Chebyshev transformer has $\mathrm{N}$ steps, the highest power of the polynomial is $(\mathrm{N}-1)$. The resulting polynomial $\rho$ is equated to.

$$
\rho=\alpha(\mathrm{N}-1) \mathrm{T}_{\mathrm{N}-1}\left(\cos \theta / \cos \theta_{1}\right)=\alpha(\mathrm{N}-1) \mathrm{T}_{\mathrm{N}-1}(\mathrm{X})
$$

to evaluate the coefficients $\rho(\mathrm{M})$.

The constant $\alpha(\mathrm{N}-1)$ is found from Eq, (8), with $\theta=0$, which corresponds to a structure of zero length, or

$$
\rho=[Z(N+1)-Z(1)] /[Z(N+1)+Z(1)]=1 / 2 \ln [Z(N+1) / Z(1)],
$$

When substituted into Eq. (22), with $\theta=0$, the result is

$$
\alpha(\mathrm{N}-1)=\frac{1 / 2 \ln [\mathrm{Z}(\mathrm{N}+1) / \mathrm{Z}(1)]}{\mathrm{T}_{\mathrm{N}-1}\left(1.0 / \cos \theta_{1}\right)}
$$

V. Graves' Method and the Chebyshev Coefficients

Chebyshev coefficients are tedious to calculate, but an unwritten method, developed by Ross E. Graves at Stanford University, makes the calculations as simple as those in Pascal's Triangle for Binomial Coefficients. My thesis advisors, Donald Reynolds and Myron Swarm at Stanford, were students under Professor Graves, and enjoyed my reference to Graves Pyramid for Chebyshev Polynomials.

Graves' method is similar to Pascal's triangle for Binomial coefficients. Pascal's Triangle is made with the number 1.0 in the first row. In the second row, $1.0 \mathrm{~s}$ are placed at the left and right of the element above. In successive rows, elements are formed by adding the two elements on 
the left and right of the calculated element in the row above the element. This ritual for element placement is described because a similar ritual is followed with Graves' Pyramid.

Graves' Pyramid is formed by inserting a number 2.0 in the first row. In the second row, another number, $\mathrm{X}_{0}=1.0 / \cos \theta_{1}$, is placed to the left of the element above and the same number, $\mathrm{X}_{0}$, is placed to the right of the number above. In successive rows, elements are formed by adding the two elements on the right and on the left in the row above, multiplying the sum by $\mathrm{X}_{0}$, and subtracting the element in the second row above the entry being calculated from this product. When elements are absent, they are assumed to be zero. Graves' Pyramid appears in Table II as a function of $\mathrm{X}_{0}$. When $\mathrm{X}_{0}=2.00$, Graves' Pyramid has the values seen in Table III. When elements are normalized we have the reflection coefficient ratios in Table IV for 10 sections or steps and Bandwidth $\mathrm{BW}=2.00$.

Table II

Graves' Pyramid for Chebyshev Coefficients

\begin{tabular}{|c|c|c|c|c|c|}
\hline & & & 2 & & \\
\hline $\mathrm{X}_{0}{ }^{3}$ & $\begin{array}{l}\mathrm{X}_{0}^{2} \\
3 \mathrm{X}_{0}{ }^{3}\end{array}$ & $\begin{array}{l}\mathrm{X}_{0} \\
2 \mathrm{X}_{0}{ }^{2} \\
-3 \mathrm{X}_{0}\end{array}$ & $\begin{array}{c}\mathrm{X}_{0} \\
-3 \mathrm{X}_{0}{ }^{3}\end{array}$ & $\begin{array}{c}\mathrm{X}_{0}{ }^{2} \\
-3 \mathrm{X}_{0}\end{array}$ & $\mathrm{X}_{0}{ }^{3}$ \\
\hline
\end{tabular}

Table III is Graves' Pyramid for Chebyshev Coefficients, $\mathrm{X}_{0}=2.00$.

Table III

Graves' Pyramid for Chebyshev Coefficients, $\mathrm{X}_{0}=2.00$

$\begin{array}{llllllllll} & & & & & 2 & & & & \\ & & & 2 & & 2 & & & \\ & & & 4 & & 6 & & 4 & & \\ & & & & 18 & & 18 & & 8 & \\ 16 & & 48 & & 66 & & 48 & & 16\end{array}$

Proceedings of the 2001 American Society for Engineering Education Annual Conference \&Exposition Copyright O 2001, American Society for Engineering Education 


\title{
Table IV
}

Graves' Pyramid for Normalized Chebyshev Coefficients, $\mathrm{X}_{0}=2.00$

Normalized by dividing elements in each row by end elements in the row.

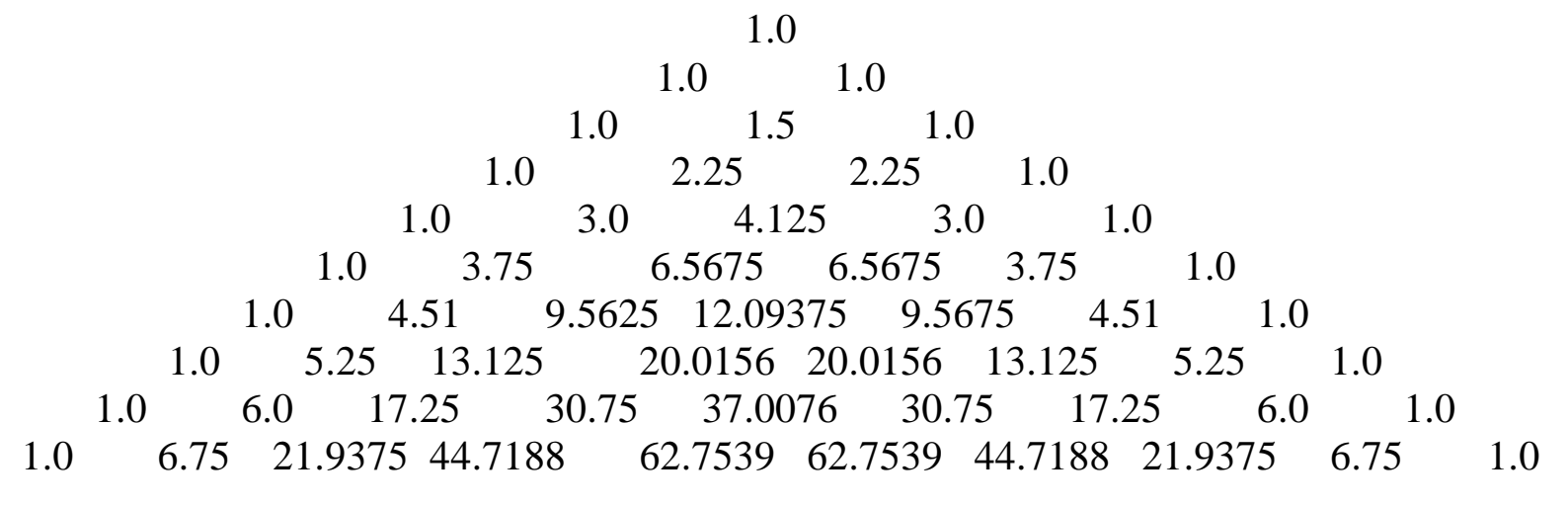

With Graves' Pyramid, the ratios of reflection coefficients to design a Chebyshev transformer for coupling slow to fast wave transmission lines for ten steps in transitions from slow to fast wave lines medical lasers and traveling wave tubes..

$$
\begin{aligned}
& \rho(1): \rho(2): \rho(3): \rho(4): \rho(5): \rho(6): \rho(7): \rho(8): \rho(9): \rho(10)= \\
& \quad 1.0: 6.75: 21.9375: 44.7188: 62.7539: 62.7539: 44.7188: 21.9375: 6.75: 1.0
\end{aligned}
$$

Bibliography

1. Cohn, Seymour N. "Optimum design of stepped transmission line transformers," IRE Trans. Microwave Theory Tech., vol. MTT-3, pp.16-21, April 1955.

2. Dolph, C.L. "A current distribution for broadside arrays which optimize the relationship between beam width and side-lobe level," Proc. IRE, vol. 34, pp. 335-348, June 1946.

3. Biggs, A. W. "Slow wave transmission line transformers." IEEE Trans. EMC, vol. 34, pp. 242-251, Aug. 1993.

\begin{abstract}
ALBERT W. BIGGS
Albert W. Biggs is a Professor of Electrical and Computer Engineering and Associate Dean at the University of Alabama at Huntsville. He is on extended Sabbatical Leave to work as a temporary Congressional Staffer with the US House when an opening is available. Dr. Biggs is a registered Professional Electrical Engineer in Kansas and is working in locating landmines with hand held and airborne radars. Dr. Biggs received a BS in Electrical Engineering from Washington University, St. Louis, Missouri, an MBA from Stanford University, Palo Alto, California, and a Ph.D. in Electrical Engineering from the University of Washington, Seattle, Washington. He has partially completed the requirements for the MD at the University of Louisville, Kentucky.
\end{abstract}

Proceedings of the 2001 American Society for Engineering Education Annual Conference \&Exposition Copyright O 2001, American Society for Engineering Education 